\title{
A randomized controlled parallel study of nalbuphine and fentanyl on hemodynamic response to laryngoscopic and laparoscopic stress in patients undergoing laparoscopic appendectomy under general anaesthesia
}

\author{
Madhu $\mathrm{S}^{1, *}$, Balarama Reddy $\mathbf{P}^{2}$, Vikas P Savdi ${ }^{3}$, Ramadas K T ${ }^{4}$ \\ ${ }^{1,2,3}$ Assistant Professor, ${ }^{4}$ Professor and HOD, Dept. of Anaesthesiology, P K Das Institute of Medical Science, Palakkad, Kerala, \\ India
}

*Corresponding Author: Madhu S

Email: madhu.asth@gmail.com

Received: $23^{\text {rd }}$ July, 2018

Accepted: $2^{\text {nd }}$ August, 2018

\begin{abstract}
Aims: Hemodynamic changes caused by laryngoscopy, endotrachial intubation and creation of pneumoperitonium in laparoscopic surgeries is deleterious to patients. This study was done to compare nalbuphine an agonist antagonist opioid with fentanyl, a gold standard opioid, when used in patients undergoing laparoscopic appendectomy under general anesthesia.

Materials and Methods: This study included 60 ASA I patients who underwent elective appendectomy were randomised to receive either nalbuphine $0.1 \mathrm{mg} / \mathrm{kg}$ or fentanyl $2 \mu \mathrm{g} / \mathrm{kg}$ as analgesics 5 minutes prior to intubation. Heart rate, systolic blood pressure, diastolic blood pressure and mean arterial pressure were recorded at baseline and at every 2 minutes post intubation till the end of surgery. The time of laparoscopic port insertion and creation of pneumoperitonium was noted. Independent student ${ }^{\prime} t$ ' test and chi square test was used to analyse continuous variables and categorical variables respectively.

Results: The changes in heart rate were comparable between both groups at all time points of observation. Nalbuphine and fentanyl group showed an increase in heart rate of $3.81 \%$ and $6.03 \%$ respectively. Mean arterial pressure was comparable at all time points of observation except at the time of insertion of second port wherein fentanyl group showed $12.70 \%$ increase as compared to $4.54 \%$ fall from baseline in nalbuphine group. Side effects were comparable between both groups except sedation which was significantly more in nalbuphine group and pruritus more in fentanyl group.

Conclusion: Nalbuphine due to its availability without license is an effective alternative to fentanyl in laparoscopic surgeries.
\end{abstract}

Keywords: Nalbuphine, Fentanyl, Hemodynamic response, Laparoscopic surgery.

\section{Introduction}

General anaesthesia, direct laryngoscopy, tracheal intubation and pneumoperitonium created in laparoscopic surgery induce marked cardiovascular changes. A rise in plasma concentration of noradrenaline, adrenaline and dopamine is known to increase heart rate and blood pressure during endotrachial intubation. Though, the responses of blood pressure and heart rate is transient, it might have detrimental effect in patients with pre-existing cardiovascular disease. ${ }^{1-4}$ In laparoscopic surgeries creation of pneumoperitonium causes hemodynamic changes by both mechanical and neurohumoral factors. ${ }^{5-}$

7 Catecholamines, renin and vasopressin have been proposed for these changes.

Various drugs and induction agents like fentanyl, remifentanyl, morphine, buprinorphine, propofol, magnesium, esmolol, clonidine, lignocaine, dexmeditomidine etc have been used for attenuation of stress response. The adverse effect profile, duration of action, and availability of these drugs guides its usage in various clinical setting. ${ }^{8-16}$

Fentanyl, a potent opioid introduced in 1960 is a gold standard in intra operative analgesia replacing morphine and pethidine because of its short duration of action, cardiac stability and minimal respiratory depression. ${ }^{17}$ Nalbuphine is an opioid agonist-antagonist used as analgesic. Its analgesic potency is equivalent to that of morphine on a milligram basis. ${ }^{18}$ It is agonist at kappa receptor and antagonists at mu receptors. It has fewer side effects as compared to other agonist antagonists such as pentazocine or butarphenol. Its cardiovascular stability, less nausea vomiting and longer duration of action makes it an ideal perioperative analgesic. ${ }^{18}$ In this study we compared nalbuphine and fentanyl for its hemodynamic effect during laryngoscopy and creation of pneumoperitonium in patients undergoing elective laparoscopic appendectomy.

\section{Materials and Methods}

After obtaining Institutional ethics committee approval, 60 patients of ASA grade 1 was included in the study after obtaining informed consent. Patients aged 20 60 , posted for elective laparoscopic appendectomy under general anaesthesia were included in the study. Patients refusing to participate in the study, ASA II and above, emergency surgeries, pregnant female, known allergy to study drugs and patients with difficult airway were excluded from the study.

A careful pre-anaesthetic evaluation was done prior to surgery and necessary investigation was done before enrolling into the study. Eligible patients who gave consent were kept nil orally for more than 6 hours before surgery. On the day of surgery all patients received premedication (glycopyrrolate $0.2 \mathrm{mg}$, and ondansetron $4 \mathrm{mg}$ intravenous) in pre operative room. In the operative 
room patients were attached with monitors (ECG, NIBP and pulse oximeter) and baseline reading was recorded. Based on randomization table generated prior, patients were divided into two groups. Group I received Nalbuphine $0.1 \mathrm{mg} / \mathrm{kg}$ and Group II received Fentanyl $2 \mu \mathrm{g} / \mathrm{kg}$ five minutes before Induction. Both group patients were induced with propofol titrated according to loss of consciousness and paralysed with atracurium 0.5 $\mathrm{mg} / \mathrm{kg}$ followed by 4 minutes of bag mask ventilation. Then the patients were intubated with appropriate endotrachial tube. Patients having unanticipated difficult airway were excluded from the study.

Heart rate, systolic blood pressure, diastolic blood pressure, mean arterial pressure, oxygen saturation (Spo2) and end tidal CO2 was recorded at baseline, 5 minutes after study drug, 1 minute after intubation, and then at 2 minutes interval till the end of surgery. Time of first port insertion, creation of pneumoperitonium, second port insertion and third port insertion was noted. Patients were maintained on $\mathrm{O} 2, \mathrm{~N} 2 \mathrm{O}$ and sevoflurane. Sevofluarne was kept at 1.5 on dial setting till appendectomy and then reduced to 1.0 with gradual tapering at the end for extubation. Inj glycopyrrolate $(0.008 \mathrm{mg} / \mathrm{kg})$ and neostigmine $0.05 \mathrm{mg} / \mathrm{kg}$ intravenously was used for reversal. Patients were monitored every 5 minutes for 30 minutes post extubation and for 6 hours in post anaesthesia care unit. Sedation in post anaesthesia care unit was assessed by Pasero Opioid - Induced sedation scale (0 -Sleep easy to arouse; 1 - Awake and alert; 2 - Slight drowsy, easily aroused; 3- Frequently drowsy, arousable, drifts off to sleep during conversation; 4 - Somnolent, minimal or no response to verbal or physical stimulation). Patients whose surgery prolonged more than 90 minutes were excluded from the study.

\section{Statistical Analysis}

The sample size 60 with 30 in each group was based on assuming a variation of 15 , a clinical acceptable difference of 10 in heart rate and systolic blood pressure, with $\alpha$ value of 0.05 and $\beta$ value of 0.20 and power of 0.80 . Independent student $t$ test was used for intergroup comparison of continuous variables and chi square test was used for comparing nominal variables. A P value of $\leq 0.05$ was considered significant.

\section{Observation and Results}

Patient demographic data was comparable between groups (Table 1). There was no significant difference in heart rate, systolic blood pressure, diastolic blood pressure and mean arterial pressure between both groups at baseline (Table 1). The change in Heart rate and mean arterial pressure from baseline was considered for analysis as change in systolic and diastolic pressure reflected similar changes.

There was no significant fall in heart rate, systolic blood pressure, diastolic blood pressure and mean arterial pressure in both groups 5 minutes after induction. Both groups showed increase in heart rate, $3.81 \%$ in nalbuphine group and $6.03 \%$ in fentanyl group, 1 minute after intubation but were comparable and statistically not significant. Heart rate changes were comparable between both the groups at all time points of observation. Maximum increase in heart rate was observed at the time of extubation, with nalbuphine group showing increase by $27.45 \%$ and fentanyl group $18.82 \%$. However the increase in heart rate was not statistically significant. (Table 2 and Fig. 1)

The changes in Mean arterial Pressure were comparable between both groups at all time points of observation except at the time of insertion of second port wherein Nalbuphine group showed $4.54 \%$ fall whereas fentanyl group showed $12.70 \%$ increase from baseline which was statistically significant. Both groups showed maximum rise at the time of extubation, $15.94 \%$ in nalbuphine group and $13.5 \%$ in fentanyl group, but was not statistically significant. (Table 3 and Fig. 2). Systolic and diastolic blood pressure showed similar changes (Fig. $3 \& 4$ ).

The incidence of post operative complication such as shivering, nausea and vomiting, respiratory depression, hypertension or hypotension, tachycardia or bradycardia was comparable between both groups. The incidence of pruritus was significantly more in fentanyl group as compared to nalbuphine group (Table 4). Sedation as assessed by Pasero Opioid - Induced sedation scale was significantly higher in nalbuphine group as compared to fentanyl group at 1.0, 2.0 and 6.0 hrs after extubation in post anaesthesia care unit.

Table 1: Demographic data and base line heart rate, Systolic blood pressure (SBP), Diastolic blood pressure (DBP) and Mean Arterial Pressure (MAP)

\begin{tabular}{|l|c|c|c|}
\hline & Group I $(\mathbf{n}=\mathbf{3 0})$ & Group II $(\mathbf{n}=\mathbf{3 0})$ & P value \\
\hline Age & $26.88 \pm 8.76$ & $26.31 \pm 8.23$ & 0.88 \\
\hline Gender & $\begin{array}{c}\text { Female }-18 \\
\text { Male }-12\end{array}$ & $\begin{array}{c}\text { Female }-16 \\
\text { Male }-14\end{array}$ & 0.72 \\
\hline Weight & $52.56 \pm 6.94$ & $52.31 \pm 9.19$ & 0.92 \\
\hline ASA I & 30 & 30 & - \\
\hline Heart rate & $80.13 \pm 13.71$ & $86.00 \pm 15.64$ & 0.26 \\
\hline SBP & $126.13 \pm 12.3$ & $119.94 \pm 16.49$ & 0.23 \\
\hline DBP & $80.19 \pm 8.42$ & $76.94 \pm 15.98$ & 0.47 \\
\hline MAP & $93.5 \pm 10.21$ & $89.04 \pm 15.91$ & 0.35 \\
\hline
\end{tabular}


Table 2: Changes in heart rate from baseline at various time points

\begin{tabular}{|l|c|c|c|c|c|}
\hline & Group I $(\mathbf{n}=30)$ & & Group II $(\mathbf{n}=\mathbf{3 0})$ & & P value \\
\hline 5 min Post Induction & $-3.06 \pm 10.56$ & $\downarrow$ & $-5.19 \pm 6.66$ & $\downarrow$ & 0.50 \\
\hline post Intubation & & & & & \\
\hline 1 min & $9.00 \pm 16.07$ & $\uparrow$ & $10.06 \pm 14.81$ & $\uparrow$ & 0.84 \\
\hline $2 \mathrm{~min}$ & $6.06 \pm 13.80$ & $\uparrow$ & $5.44 \pm 14.75$ & $\uparrow$ & 0.90 \\
\hline $4 \mathrm{~min}$ & $3.38 \pm 12.50$ & $\uparrow$ & $3.13 \pm 16.29$ & $\uparrow$ & 0.96 \\
\hline $6 \mathrm{~min}$ & $2.31 \pm 12.65$ & $\uparrow$ & $3.56 \pm 13.27$ & $\uparrow$ & 0.78 \\
\hline $8 \mathrm{~min}$ & $5.13 \pm 13.97$ & $\uparrow$ & $3.56 \pm 11.31$ & $\uparrow$ & 0.73 \\
\hline $10 \mathrm{~min}$ & $6.56 \pm 13.42$ & $\uparrow$ & $3.38 \pm 12.09$ & $\uparrow$ & 0.48 \\
\hline $12 \mathrm{~min}$ & $5.44 \pm 15.19$ & $\uparrow$ & $5.25 \pm 13.25$ & $\uparrow$ & 0.97 \\
\hline $14 \mathrm{~min}$ & $5.56 \pm 18.08$ & $\uparrow$ & $-0.31 \pm 14.23$ & $\downarrow$ & 0.31 \\
\hline $16 \mathrm{~min}$ & $4.69 \pm 18.05$ & $\uparrow$ & $-0.13 \pm 13.16$ & $\downarrow$ & 0.39 \\
\hline First port & $5.06 \pm 14.20$ & $\uparrow$ & $7.19 \pm 12.57$ & $\uparrow$ & 0.65 \\
\hline Pneumoperitoneum & $5.38 \pm 12.52$ & $\uparrow$ & $5.31 \pm 11.28$ & $\uparrow$ & 0.98 \\
\hline Second port & $5.94 \pm 14.91$ & $\uparrow$ & $5.13 \pm 13.76$ & $\uparrow$ & 0.87 \\
\hline Third port & $6.94 \pm 16.80$ & $\uparrow$ & $2.75 \pm 13.95$ & $\uparrow$ & 0.45 \\
\hline Extubation & $22.00 \pm 17.10$ & $\uparrow$ & $16.19 \pm 14.30$ & $\uparrow$ & 0.30 \\
\hline 5 min post extubation & $7.19 \pm 19.08$ & $\uparrow$ & $9.75 \pm 16.22$ & $\uparrow$ & 0.68 \\
\hline
\end{tabular}

Table 3: Changes in Mean Arterial Pressure from baseline at various time points $(\downarrow$ - decrease from base line; $\uparrow$ Increase from baseline)

\begin{tabular}{|l|c|c|c|c|c|}
\hline & Group I (n=30) & Change & Group II (n=30) & & P value \\
\hline 5 min Post Induction & $-20.15 \pm 14.02$ & $\downarrow$ & $-18.95 \pm 14.20$ & $\downarrow$ & 0.81 \\
\hline post Intubation & & & & & \\
\hline $1 \mathrm{~min}$ & $2.37 \pm 18.01$ & $\uparrow$ & $1.50 \pm 22.04$ & $\uparrow$ & 0.90 \\
\hline $2 \mathrm{~min}$ & $-8.00 \pm 12.61$ & $\downarrow$ & $-5.06 \pm 20.64$ & $\downarrow$ & 0.63 \\
\hline $4 \mathrm{~min}$ & $-16.18 \pm 12.89$ & $\downarrow$ & $-5.37 \pm 21.45$ & $\downarrow$ & 0.09 \\
\hline $6 \mathrm{~min}$ & $-16.87 \pm 11.85$ & $\downarrow$ & $-3.64 \pm 22.54$ & $\downarrow$ & 0.04 \\
\hline $8 \mathrm{~min}$ & $-7.73 \pm 17.94$ & $\downarrow$ & $2.14 \pm 22.07$ & $\uparrow$ & 0.17 \\
\hline $10 \mathrm{~min}$ & $-2.68 \pm 15.13$ & $\downarrow$ & $6.44 \pm 20.14$ & $\uparrow$ & 0.15 \\
\hline $12 \mathrm{~min}$ & $-2.81 \pm 15.46$ & $\downarrow$ & $7.25 \pm 14.78$ & $\uparrow$ & 0.07 \\
\hline $14 \mathrm{~min}$ & $0.43 \pm 17.11$ & $\uparrow$ & $3.45 \pm 19.79$ & & 0.64 \\
\hline $16 \mathrm{~min}$ & $-0.48 \pm 15.15$ & $\downarrow$ & $0.92 \pm 17.87$ & $\uparrow$ & 0.81 \\
\hline First port & $-7.41 \pm 15.08$ & $\downarrow$ & $4.14 \pm 20.07$ & $\uparrow$ & 0.07 \\
\hline Pneumoperitoneum & $-3.72 \pm 15.96$ & $\downarrow$ & $-9.08 \pm 23.72$ & $\downarrow$ & 0.08 \\
\hline Second port & $-4.25 \pm 13.15$ & $\downarrow$ & $11.31 \pm 20.32$ & $\uparrow$ & 0.01 \\
\hline Third port & $-2.50 \pm 16.40$ & $\downarrow$ & $6.52 \pm 18.87$ & $\uparrow$ & 0.16 \\
\hline Extubation & $14.91 \pm 15.25$ & $\uparrow$ & $12.08 \pm 14.76$ & $\uparrow$ & 0.59 \\
\hline 5 min post extubation & $5.16 \pm 13.70$ & $\uparrow$ & $9.41 \pm 17.29$ & $\uparrow$ & 0.45 \\
\hline
\end{tabular}

Table 4: Side effects in Post operative period

\begin{tabular}{|l|c|c|c|}
\hline & Group I (n=30) & Group II (n=30) & P value \\
\hline Shivering & $4 / 30$ & $8 / 30$ & 0.36 \\
\hline Pruritus & $0 / 30$ & $14 / 30$ & 0.007 \\
\hline Nausea/Vomiting & Nil & Nil & \\
\hline Bradycardia/tachycardia & Nil & Nil & \\
\hline Hypotension/hypertension & Nil & Nil & \\
\hline Sedation $(1 \mathrm{hr})$ & $3.0 \pm 0.52$ & $2.25 \pm 0.45$ & 0.001 \\
\hline Sedation $(2 \mathrm{hr})$ & $2.06 \pm 0.25$ & $1.69 \pm 0.48$ & 0.01 \\
\hline Sedation $(6 \mathrm{hr})$ & $1.94 \pm 0.25$ & $1.0 \pm 0.1$ & 0.001 \\
\hline
\end{tabular}




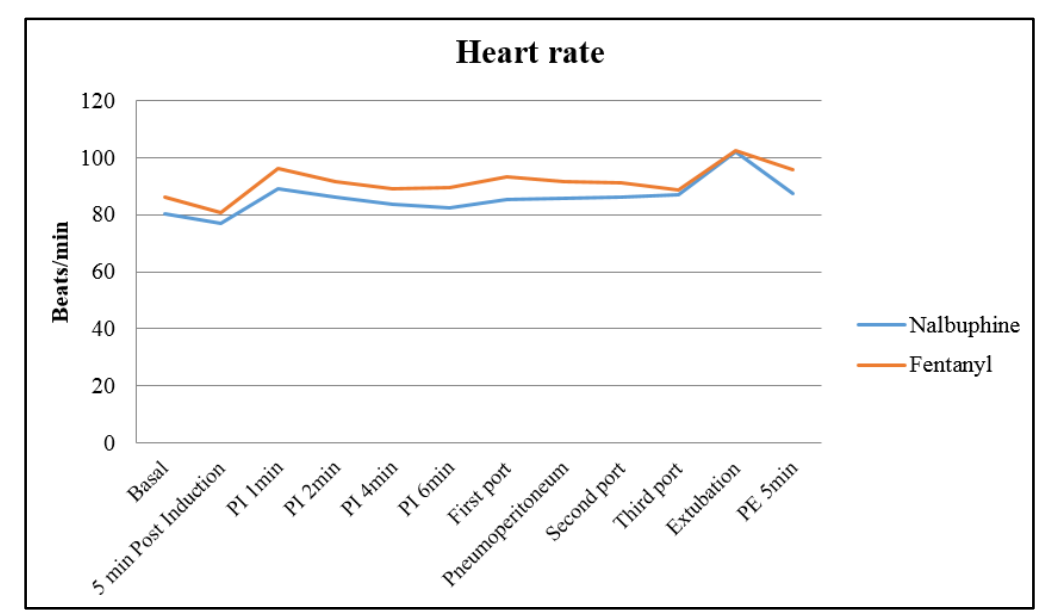

Fig. 1: Changes in heart rate at various time points (PI: Post Intubation).

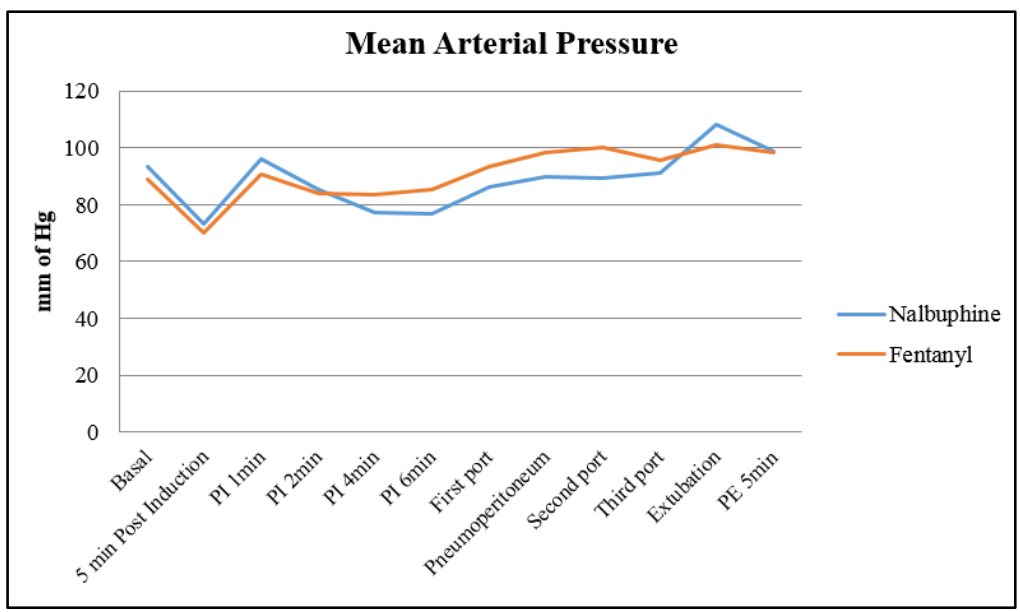

Fig. 2: Changes in mean arterial pressure at various time points (PI: Post Intubation)

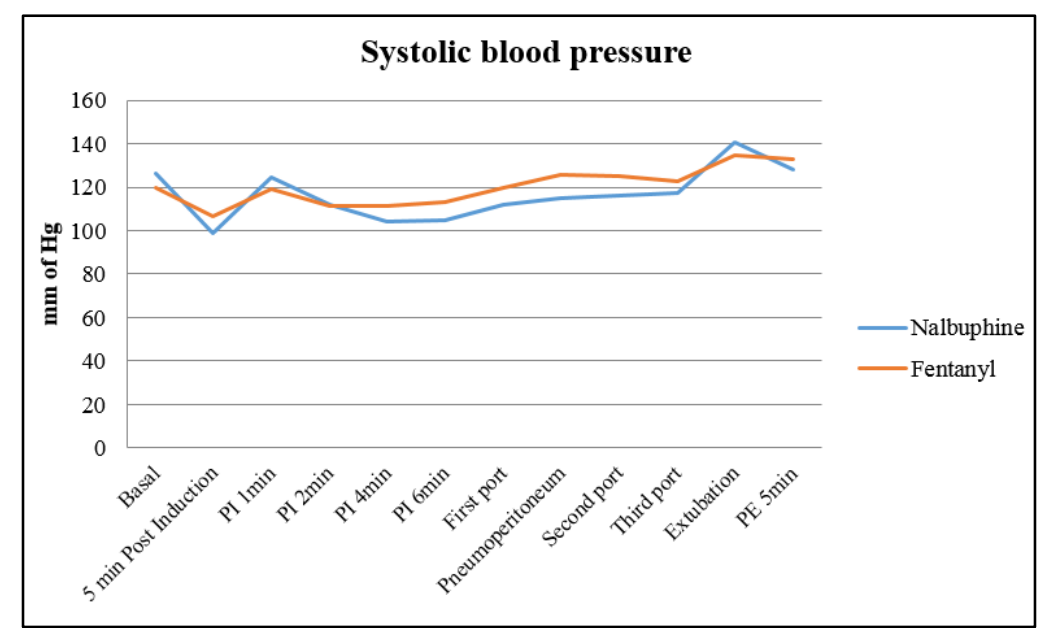

Fig. 3: Changes in systolic blood pressure at various time points (PI: Post Intubation). 


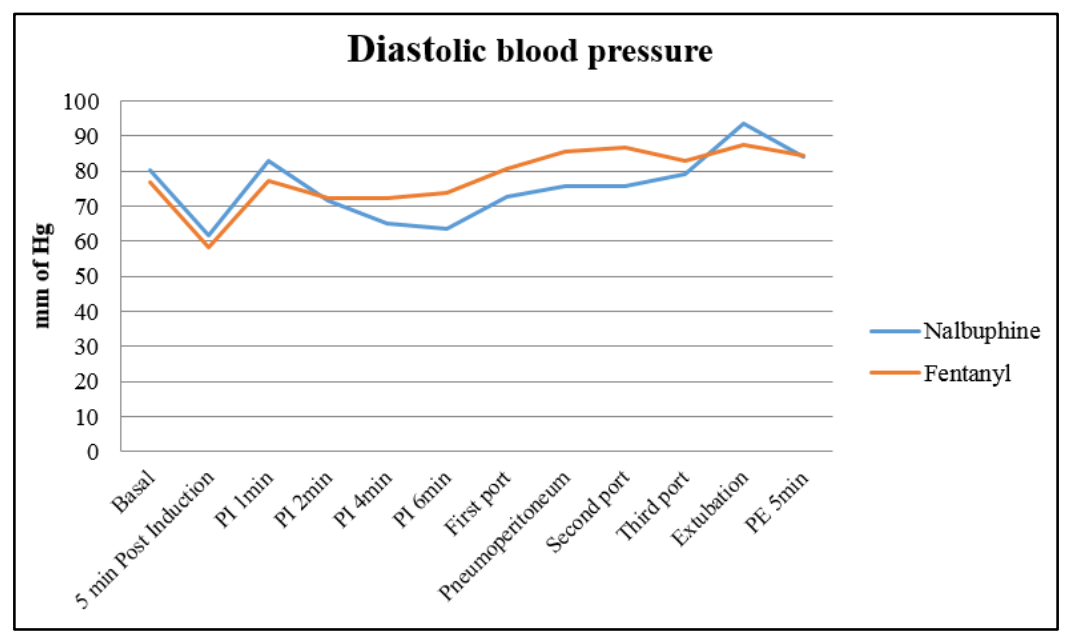

Fig. 4: Changes in diastolic blood pressure at various time points (PI: Post Intubation)

\section{Discussion}

The main objective of balanced anaesthesia is to provide amnesia, minimize pain and reduce the adverse effects associated with agents used for anaesthesia. The components of balanced anaesthesia include amnesia, analgesia, muscle relaxation and abolition of autonomic reflexes. ${ }^{19}$ Opioids has been a mainstay analgesic in balanced anaesthesia since 1947. The advantages of opioids are less fluctuation in cardiovascular dynamics, decrease requirements of inhalation anaesthetics and increased post operative analgesia. ${ }^{19}$ The use of opioids is limited by its adverse effect such as bradycardia, hypotension, respiratory depression and its availability. Because of its abuse potential opioid availability is strictly regulated by state.

Nalbuphine, is now commercially available in India and can be obtained without an opioid liscence. Nalbuphine has a comparable analgesic potential to morphine as demonstrated in the study done by Joseph Yanulevich ${ }^{20,21}$ Unlike morphine, nalbuphine has a plateau effect on respiratory depression and also has been used to reverse the respiratory depression due to morphine. ${ }^{22}$ This study aimed at comparing effect of nalbuphine on hemodynamics with that of fentanyl, a gold standard opioid in India, in laparoscopic surgeries. Earlier studies comparing nalbuphine with fentanyl for hemodynamic response have used $0.2 \mathrm{mg} / \mathrm{kg}-0.3 \mathrm{mg} / \mathrm{kg}$ of nalbuphine ${ }^{14,23-25} \mathrm{In}$ our study we used $0.1 \mathrm{mg} / \mathrm{kg}$ of nalbuphine as nalbuphine is equipotent to morphine in milligram basis and also to minimise postoperative side effects of nalbuphine such as sedation, shivering, dizziness/vertigo and euphoria. ${ }^{26}$

In patients undergoing laparoscopic surgeries under general anesthesia, laryngoscopy and intubation are not the only manoeuvres which cause increase in heart rate and blood pressure but also insertion of laparoscopic ports and creation of pneumoperitonium ${ }^{5,7}$ This study compared nalbuphine and fentanyl on its hemodynamic effects during these manoeuvres.
In our study, heart rate was comparable between both groups at all time points of observation. The maximum increase in heart rate post intubation was seen at 1 minute with nalbuphine showing $11.23 \%$ and fentanyl group showing $11.69 \%$. Khan et al found a significant increase in heart rate $(25 \%)$ in nalbuphine group as compared to fentanyl group (6.4\%). This may be attributed to selection of patients and use of TIVA. ${ }^{26}$

At the time of intubation, mean arterial pressure showed an increase by $2.53 \%$ in nalbuphine group as compared to $1.68 \%$ in fentanyl group in our study. Tariq et $a l^{5}$ in their study comparing nalbuphine and saline for endotrachial intubation, observed $1.40 \%$ increase in MAP at 1 min post intubation. The difference may be due to higher dose $(0.2 \mathrm{mg} / \mathrm{kg})$ used in their study.

The cardiovascular response to increased abdominal pressure is phasic and transient. However, this can lead to serious complication in patients with cardiac or respiratory diseases. Hence evaluation of effect of drugs used to attenuate stress response during insertion of laparoscopic port and creation of pneumoperitonium is essential. We found in our study that heart rate was comparable between both groups during first port insertion, creation of pneumoperitonium, second port insertion and third port insertion. Nalbuphine group showed $6.7 \%$ increase in heart rate after pneumoperitonium as compared to $6.17 \%$ in fentanyl group. However, mean arterial pressure showed a significant increase in fentanyl group $12.70 \%$ as compared to $4.5 \%$ fall from baseline in nalbuphine group. These changes were also reflected in both systolic and diastolic blood pressure. These finding may be due to patient group selected in our study which included patients posted for elective laparoscopic appendectomy so as to control patient and surgery related variable factor in evaluation of drug effect. Further studies are required in various other surgeries to evaluate these findings.

At the time of extubation we observed comparable increase in both heart rate and mean arterial pressure in both groups in contrast to observation by Khan et al. In 
their study they observed $15 \%$ increases in heart rate in nalbuphine group as compared to $4 \%$ in fentanyl group. This may be due to additional supplementation of analgesic in fentanyl group after 1 hour of surgery in their study. In our study, fentanyl group additionally received Inj paracetamol $1 \mathrm{gm}$ intravenous after 45 minutes of induction. However, the average duration of surgery in both groups was less than 1 hour. Laparoscopic appendectomy was hence chosen for this study so as to control the duration of surgery.

In the post operative ward the incidence of shivering was more in nalbuphine group as compared to fentanyl group. The requirement of additional analgesic in first 6 hours of post operative observation was more in fentanyl group as compared to nalbuphine group. This might be due to longer duration of action of nalbuphine. However, further controlled studies are required to evaluate post operative analgesic effect of nalbuphine.

In our study, we included surgeries which could get over in one hour duration; further studies are required in longer duration and emergency surgeries. Recovery from anaesthesia was not objectively evaluated in our study, this may one of the limitation of our study as nalbuphine is known to cause more sedation than fentanyl. ${ }^{26}$

\section{Conclusion}

Thus, we conclude that nalbuphine at dose of $0.1 \mathrm{mg} / \mathrm{kg}$ is an effective alternative for fentanyl in laparoscopic appendectomy surgeries. Due to its availability without opioid licence makes it a better alternative for fentanyl at small hospitals.

\section{Conflict of Interest: None}

\section{References}

1. Siedleckj. I Disturbances in the function of cardiovascular system in patients following endotracheal intubation and attempts to their prevention by pharmacological blockade of the sympathetic system. Anaesthesia, Resuscitation and Intensive Therapy 1975;3:107-123.

2. Russell WJ, Morris RG, Frewin D B. Changes in plasma catecholamines concentration during endotracheal intubation. Br J Anaesth. 1981;53:837-839.

3. Fox Elizabeth J, Garry S, Hill Constance H, Villanveva Raymond, King Benton D. Complications Related to the Pressor Response to Endotracheal Intubation. Anesthesiology. 1977;44:524-525.

4. Lindgren L, Yli Hankala A, Randell T, M Kirvela, M Scheinin, PJ Neuvonen. Haemodynamic and catecholamine responses to induction of anaesthesia and tracheal intubation: comparison between propofol and thiopentone. Br J Anaesth. 1993;70:306-310.

5. Wahba RWM, Beique F, Kleiman SJ. Cardiopulmonary function and laparoscopic cholecystectomy. Can J Anaesth. 1995;42:51- 63.

6. Sharma KC, Brandstetter RD, Brensilver JM, Jung LD. Cardiopulmonary physiology and pathophysiology as a consequence of laparoscopic surgery. Chest. 1996; 110:810-815.

7. Joris JL, Noirot DP, Legrand MJ, Jacquet NJ, Lamy ML. Hemodynamic changes during laparoscopic cholecystectomy. Anesth Analg. 1993;76:1067-1071.
8. Maguire M, Kumar N, Parker JL, Rowbotham DJ, Thompson JP. Comparison of effects of remifentanil and alfentanil on the cardiovascular response to tracheal intubation in hypertensive patients. Br J Anaesth. 2001;86:90-93.

9. Martineau RJ, Tousignant CP, Miller DR. Alfentanil controls the hemodynamic response during rapid sequence induction of anaesthesia. Can J Anaesth. 1990;37:755-761.

10. FA Khan, RS Kamal. Effect of Buprenorphine on the cardiovascular response to tracheal intubation. Anaesthesia. 1989;44:394-397.

11. Jain PN, Divatia JV, Manjshree SS, Chatopadhyay G, Shah SC. Intravenous magnesium inhibits pressure response to nasotracheal intubation. J Anaesth Clin Pharmacol. 1995;11:59-62.

12. Minal FN, Khan FA. A comparision of morphine and nalbuphine for intraoperative and postoperative analgesia. J Pak Med Assoc. 2003;53:391-396.

13. Beaver WT, Felse GA. A comparison of the analgesic effect of intramuscular nalbuphine and morphine in patients with postoperative pain. J Pharmacol Exp Ther. 1978;204:486-496.

14. Khan FA, Hameedullah. Comparison of fentanyl and nalbuphine in total intravenous anaesthesia (TIVA). $J$ Pak Med Assoc. 2002;52:459-465

15. Soltani Mohammadi S, Maziar A, Saliminia A. Comparing Clonidine and Lidocaine on Attenuation of Hemodynamic Responses to Laryngoscopy and Tracheal Intubation in Controlled Hypertensive Patients: A Randomized, Double-Blinded Clinical Trial. Anesthesiology and Pain Medicine. 2016;6(2):e34271. doi:10.5812/aapm.34271.

16. Patel CR, Engineer SR, Shah BJ, Madhu S. The effect of dexmedetomidine continuous infusion as an adjuvant to general anesthesia on sevoflurane requirements: A study based on entropy analysis. J Anaesthesiol Clin Pharmacol. 2013;29:318-322.

17. Shribman AJ, Smith G, Achola KJ. Cardiovascular and catecholamine response to laryngoscopy with and without tracheal intubation [abstract]. Br J Anaesth. 1987;59:295299.

18. Zaigmond EK, Winime AP, Raza SMA. Nalbuphine as an analgesic component in balanced anesthesia for cardiac surgery. Anesth. Analg. 1987;66:1155-1164.

19. Bovill JG, Sebel PS, Stanley TH. Opioid analgesics in anesthesia: with special reference to their use in cardiovascular anesthesia. Anesthesiology. 1984;61(6):731-755.

20. Joseph Yanulevich. Outpatient anesthesia with nalbuphine hydrochloride. AANA Journal August. 1983;1:395-397.

21. Oliveira ARD, Weston AC, Martinelli ES. May We Consider the Use of Nalbuphine as Postoperative Opioid in Bariatric Patients? J Anesth Crit Care Open Access. 2016;4:00159.

22. Baxter, A. D., Samson, B., Penning, J., Doran, R. and Dube, L. M. Prevention of epidural morphine-induced respiratory depression with intravenous nalbuphine infusion in post-thoracotomy patients. Can J Anaesth. 1989;36:503-509.

23. Weiss BM1, Schmid ER, Gattiker RI. Comparison of nalbuphine and fentanyl anesthesia for coronary artery bypass surgery. Hemodynamics, hormonal response, and postoperative respiratory depression. Anesth Analg. 1991;73:521-529. 
24. Neha Sharma, Hetal Parikh. A comparative study of hemodynamic responses to intubation: fentanyl versus nalbuphine Gujarat Medical Journal. 2014;69:48-53.

25. Ossama Hamdy Salman A controlled, double blind, study of adding Nalbuphine to Propofol for laryngeal mask insertion conditions and hemodynamics in adults. Egypt $J$ of Anaesth. 2015;31(4):277-281.

26. Pugh GC, Drummond GB. A dose response study with nalbuphine hydrochloride for pain in patients after upper abdominal surgery. Br J Anaesth. 1987;59:1356-1363.

How to cite this article: Madhu S, Balarama R P, Savdi V P, Ramadas K T. A randomized controlled parallel study of nalbuphine and fentanyl on hemodynamic response to laryngoscopic and laparoscopic stress in patients undergoing laparoscopic appendectomy under general anaesthesia. Indian $\mathbf{J}$ Clin Anaesth. 2018;5(4):505-511. 